\title{
Trajectory Design for Mechanical Control Systems: from Geometry to Algorithms
}

\author{
Francesco Bullo \\ Mechanical and Environmental Engineering \\ University of California at Santa Barbara \\ Santa Barbara, CA 93106 \\ bullo@engineering.ucsb.edu, http://www.me.ucsb.edu/bullo
}

European Journal of Control, Vol 10:5, pages 397-410, 2004

Special Issue on "Lagrangian and Hamiltonian Methods for Nonlinear Control"

This version compiled on September 7, 2007

\begin{abstract}
Trajectory design is a key problem in a vast array of robotic applications including the design of autonomous agile vehicles and of minimalist manipulators. This problem can be accurately formalized within the language of affine connections and of geometric control theory. This paper surveys recent results on kinematic controllability and on oscillatory controls. The treatment emphasizes how to translate geometric controllability conditions into algorithms for generating and tracking trajectories.
\end{abstract}

Keywords: mechanical systems, trajectory planning, controllability, differential geometric methods, robotics

\section{Introduction}

\section{Problem description and motivation}

One of the most fundamental capabilities for an autonomous or semi-autonomous robotic system is the ability to quickly plan and reliably execute its own motions. This low-level primitive is crucial for any high-level robot programming and controlling system. Unfortunately, motion planning and trajectory optimization problems are generically non-convex and have exponential complexity in the accuracy of the system and environment model.

This paper addresses trajectory analysis and design problems for dynamical systems with Lagrangian structure. This structure is shared by (i) robotic manipulators and mobile platforms, (ii) vehicles in space, land, air, sea and other environments, and (iii) mechanisms that locomote exploiting nonholonomic constraints and impacts (e.g., snake-like and legged robots). For such systems, typical motion planning challenges include underactuation (fewer control inputs than degrees of freedom) and complex nonlinear dynamics. This work is further motivated by the scientific interest and by the unifying mathematical language underlying geometric mechanics and geometric control theory.

\section{Paper organization and literature review}

This survey paper describes various geometric structures of mechanical systems and their relevance in trajectory design problems. The paper presents a subset of the material developed in the upcoming text [12]. This text presents a comprehensive collection of modeling, analysis and design results based on Riemannian geometry tools. Central to the presentation is the notion of affine connections and its computational implications. The presentation in this article is inspired by and relies in part on the recent results in $[4,6,7,17,30,33,35]$.

The classic structure of mechanical systems exploited in stabilization problems is passivity. Indeed, numerous important control problems rely in their essence on the existence of a total energy function and its use as a candidate Lyapunov function, see for example [3, 43, 50]. In contrast, this paper focuses on different geometric properties of mechanical systems including homogeneity, controllability, kinematic reductions, symmetry, and response to oscillatory controls.

Section 2 presents affine connection models of simple mechanical control systems subject to constraints. Most concepts we describe are standard; e.g., see [1]. The interest in Riemannian geometry concepts in the study of mechanical control systems can be seen for example in $[7,19,23,33]$. Regarding the modeling of nonholo- 
nomic systems, we follow the treatment in [30]. Symmetric product and geodesic invariance were originally introduced in [19, 29].

The modeling results are important in the study of novel biomimetic locomotion systems. Example devices include the snakeboard $[34,45]$, the $G$-snakes and roller racer models in [26, 27], the roller-walker [21], carangiform [25] and eel robots [18].

Section 3 introduces and characterizes the notion of kinematic reductions as a reduced-order modeling technique adapted to simple mechanical control systems. The treatment follows the lines of $[11,13]$, and was originally motivated in $[2,35]$.

Section 4 reviews the important results of [33] on controllability and configuration controllability. We include a catalog of prototype example systems characterized by their controllability properties and kinematic reductions.

Section 5 presents an averaging analysis for mechanical control systems subject to oscillatory input forces, i.e., input forces of large amplitude and high frequency. Building on classic averaging theory we characterize the averaged system as a simple mechanical control system. The treatment is taken from $[9,38]$. Our treatment also naturally leads to the notion of averaged potential as a means to characterize the average behavior; see [4, 24].

Section 6 presents trajectory design algorithms for controllable kinematic reductions of mechanical control systems. In other words, we design motion plans for kinematic models that can switch between specified sets of admissible vector fields. Note that the literature on inverse kinematics suggests numerous techniques, including the inverse kinematics approach described in [41], and the general polynomial programming approach in [36]. Since no general treatment can lead to analytic closedform expressions, this section discusses an interesting example system from [39].

Section 7 presents trajectory design algorithms that rely on oscillatory controls and an approximate inversion algorithm. This work was originally presented in [38] and is inspired by numerous previous works on such matters including for example $[28,48]$.

\section{Acknowledgments}

This work would not have been possible without years of fruitful collaboration with Andrew D. Lewis, the initial support and encouragement of Richard M. Murray, and the numerous key contributions by Jorge Cortés, Kevin M. Lynch, Sonia Martínez, and Miloš Žefran. This work was supported by the U.S. Army Research Office Grant DAAD 190110716 and NSF Grant IIS-0118146.

\section{Models of Mechanical Systems}

In this section we review a modeling framework for mechanical control systems. We consider the class of simple mechanical control systems with constraints, that is, systems whose total energy is the sum of kinetic and potential energy. This class includes multi-body manipulators, aerospace and underwater vehicles, and mobile mechanisms; see Figure 1. We model these systems as affine connection control systems, and study their representations in various local bases of vector fields.

\subsection{Simple mechanical control systems with constraints}

A simple mechanical control system (with constraints) is a sixtuple $\left(\mathrm{Q}, \mathbb{G}, V, F_{\text {diss }}, \mathcal{D}, \mathcal{F}\right)$ comprised of the following objects:

(i) an $n$-dimensional configuration manifold $\mathrm{Q}$,

(ii) a Riemannian metric $\mathbb{G}$ on $Q$ describing the kinetic energy,

(iii) a function $V$ on $\mathrm{Q}$ describing the potential energy,

(iv) a dissipative force $F_{\text {diss }}$,

(v) a distribution $\mathcal{D}$ of feasible velocities describing the linear velocity constraints, and

(vi) a set of $m$ covector fields $\mathcal{F}=\left\{F^{1}, \ldots, F^{m}\right\}$ defining the control forces.

For simplicity of presentation, we shall assume all quantities to be analytic and we shall assume regularity of all relevant distributions. Given the metric $\mathbb{G}$ and the distribution $\mathcal{D}$, we define the following objects. We let $P: T Q \rightarrow T Q$ be the orthogonal projection onto the distribution $\mathcal{D}$ with respect to the metric $\mathbb{G}$. We let ${ }^{\mathbb{G}} \nabla$ be the Levi-Civita connection on $\mathrm{Q}$ induced by the metric $\mathbb{G}$. We let $\nabla$ be the constrained affine connection defined by

$$
\nabla_{X} Y={ }^{\mathbb{G}} \nabla_{X} Y-\left({ }^{\mathbb{G}} \nabla_{X} P\right)(Y),
$$

for any vector fields $X$ and $Y$. When the vector field $Y$ takes value in $\mathcal{D}$, we have

$$
\nabla_{X} Y=P\left({ }^{\mathbb{G}} \nabla_{X} Y\right) .
$$

Given the Riemannian metric $\mathbb{G}$, we let $\mathbb{G}: T \mathrm{Q} \rightarrow T^{*} \mathrm{Q}$ and $\mathbb{G}^{-1}: T^{*} \mathrm{Q} \rightarrow T \mathrm{Q}$ denote the isomorphisms associated with $\mathbb{G}$. For $a \in\{1, \ldots, m\}$, we define the input vector fields $Y_{a}=P\left(\mathbb{G}^{-1}\left(F^{a}\right)\right)$, the family of input vector fields $\mathcal{Y}=\left\{Y_{1}, \ldots, Y_{m}\right\}$, and the input distribution $y$ with $y_{q}=\operatorname{span}_{\mathbb{R}}\left\{Y_{1}(q), \ldots, Y_{m}(q)\right\}$. The gradient of the 

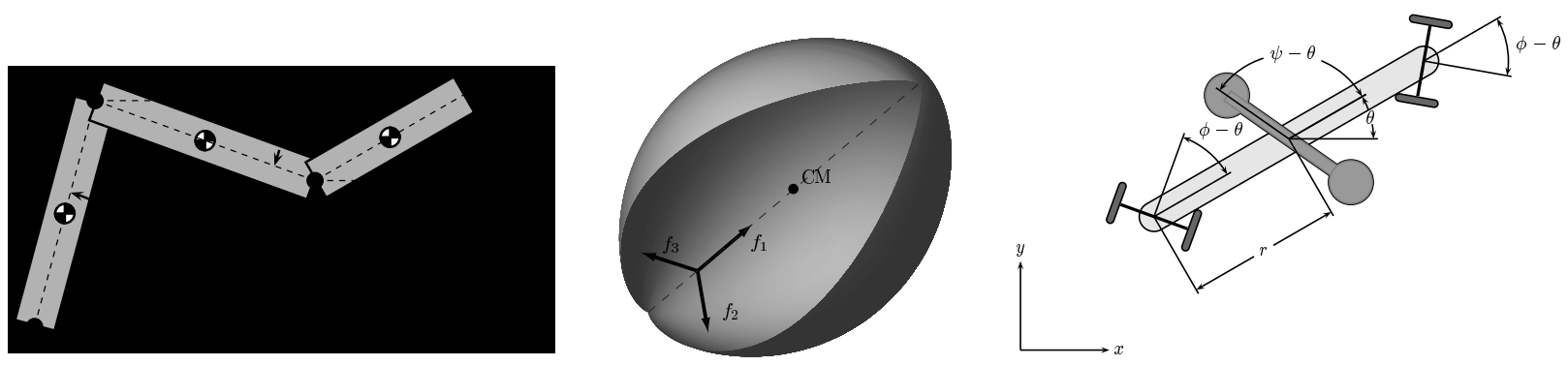

Figure 1: Simple mechanical control systems include multi-body robots, aerospace and underwater vehicles, and mobile mechanisms.

function $V$ is the vector field grad $V$ defined implicitly by $\mathbb{G}(\operatorname{grad} V, X)=\mathcal{L}_{X} V$, where $\mathcal{L}_{X} f$ is the Lie derivative of the scalar function $f$ with respect to the vector field $X$.

A controlled trajectory for the simple mechanical control system with constraints $\left(\mathrm{Q}, \mathbb{G}, V, F_{\text {diss }}, \mathcal{D}, \mathcal{F}\right)$ is a pair $(\gamma, u)$ with $\gamma:[0, T] \rightarrow Q$ and $u=\left(u_{1}, \ldots, u_{m}\right):[0, T] \rightarrow$ $\mathbb{R}^{m}$ satisfying the controlled Euler-Lagrange equations (sometimes also referred to as the controlled geodesic equations):

$$
\begin{aligned}
& \nabla_{\gamma^{\prime}(t)} \gamma^{\prime}(t)=-P(\operatorname{grad} V(\gamma(t))) \\
& +P\left(\mathbb{G}^{-1}\left(F_{\mathrm{diss}}\left(\gamma^{\prime}(t)\right)\right)\right)+\sum_{a=1}^{m} Y_{a}(\gamma(t)) u_{a}(t)
\end{aligned}
$$

Here we assume that $\gamma^{\prime}(0) \in \mathcal{D}_{\gamma(0)}$; this implies that $\gamma^{\prime}(t) \in \mathcal{D}_{\gamma(t)}$ for all $t \in[0, T]$. Furthermore, we assume the input functions $u=\left(u_{1}, \ldots, u_{m}\right):[0, T] \rightarrow \mathbb{R}^{m}$ take values in the set of Lebesgue measurable functions $\mathcal{U}_{\mathrm{dyn}}^{m}$.

\subsection{Coordinate representations}

On an open subset $U \subset \mathrm{Q}$ let $\mathcal{X}=\left\{X_{1}, \ldots, X_{n}\right\}$ be a basis for $T Q$. Let $\mathbb{G}_{i j}$ be the $(i, j)$ component of $\mathbb{G}$ and let $\mathbb{G}^{m k}$ be the $(m, k)$ component of $\mathbb{G}^{-1}$. We write the covariant derivative of the vector fields in the basis $\mathcal{X}$ as

$$
\nabla_{X_{i}} X_{j}=\left({ }^{\mathcal{X}} \Gamma\right)_{i j}^{k} X_{k},
$$

where the $n^{3}$ functions $\left\{\left({ }^{\mathcal{X}} \Gamma\right)_{i j}^{k} \mid i, j, k \in\{1, \ldots, n\}\right\}$ are called the generalized Christoffel symbols with respect to $\mathcal{X}$. Given vector fields $Y$ and $Z$ on $U$, we can write $Y=Y^{i} X_{i}$ and $Z=Z^{i} X_{i}$. The covariant derivative of $Z$ with respect to $Y$ is

$$
\nabla_{Y} Z=\left(\left(\mathcal{L}_{X_{i}} Z^{k}\right) Y^{i}+\left({ }^{\mathcal{X}}\right)_{i j}^{k} Z^{i} Y^{j}\right) X_{k} .
$$

It is instructive to write the controlled Euler-Lagrange equations with respect to the basis $\mathcal{X}$. Let the velocity curve $\gamma^{\prime}: I \rightarrow T U$ have components $\left(v^{1}, \ldots, v^{n}\right)$ with respect to $\mathcal{X}$, i.e.,

$$
\gamma^{\prime}(t)=v^{i}(t) X_{i}(\gamma(t))
$$

The pair $(\gamma, u)$ is a controlled trajectory for the controlled Euler-Lagrange equations (1) if and only if it solves the controlled Poincaré equations

$$
\begin{aligned}
\dot{v}^{k}+\left({ }^{\mathcal{X}} \Gamma\right)_{i j}^{k}(\gamma) v^{i} v^{j} & =-(P \operatorname{grad} V)^{k}(\gamma) \\
& +P_{l}^{k} \mathbb{G}^{l i}\left(F_{\mathrm{diss}}\right)_{i}\left(\gamma^{\prime}\right)+\sum_{a=1}^{m} Y_{a}^{k}(\gamma) u_{a} .
\end{aligned}
$$

Remark 2.1. If the distribution $\mathcal{D}$ has rank $p<n$, it is useful to construct a local basis for TQ by selecting the first $p$ vector fields to generate $\mathcal{D}$, and the remaining $n-p$ to generate $\mathcal{D}^{\perp}$. In this case, one can see that $v^{k}(t)=0$ for all time $t$ and all $k \in\{p+1, \ldots, n\}$.

Let $\left(q^{1}, \ldots, q^{n}\right)$ be a coordinate system for the open subset $U \subset \mathrm{Q}$. The curve $\gamma: I \rightarrow U$ has therefore components $\left(\gamma^{1}, \ldots, \gamma^{n}\right)$. The coordinate system on $U$ induces the natural coordinate basis $\left\{\frac{\partial}{\partial q^{1}}, \ldots, \frac{\partial}{\partial q^{n}}\right\}$ for the tangent bundle $T U$. With respect to this basis, we write the velocity curve $\gamma^{\prime}: I \rightarrow T U$ as

$$
\gamma^{\prime}(t)=\dot{\gamma}^{i}(t) \frac{\partial}{\partial q^{i}}(\gamma)
$$

In the coordinate system $\left(q^{1}, \ldots, q^{n}\right)$, we write $\gamma=$ $\left(\gamma^{1}, \ldots, \gamma^{n}\right), \gamma^{\prime}=\left(\dot{\gamma}^{1}, \ldots, \dot{\gamma}^{n}\right)$, and the controlled EulerLagrange equations read

$$
\begin{aligned}
\ddot{\gamma}^{k}+\Gamma_{i j}^{k}(\gamma) \dot{\gamma}^{i} \dot{\gamma}^{j} & =-(P \operatorname{grad} V)^{k}(\gamma) \\
& +P_{l}^{k} \mathbb{G}^{l i}\left(F_{\mathrm{diss}}\right)_{i}\left(\gamma^{\prime}\right)+\sum_{a=1}^{m} Y_{a}^{k}(\gamma) u_{a} .
\end{aligned}
$$

Here, the terms in the right-hand side and the Christoffel symbols $\left.\Gamma_{i j}^{k}, i, j, k \in\{1, \ldots, n\}\right\}$ are computed with respect to the natural coordinate basis. 


\subsection{Computational tools}

Let $[X, Y]$ be the Lie bracket between the vector fields $X$ and $Y$. Given a collection of vector fields $\mathcal{X}=$ $\left\{X_{1}, \ldots, X_{\ell}\right\}$, consider the associated distribution $X$ defined by $X_{q}=\operatorname{span}_{\mathbb{R}}\left\{X_{1}(q), \ldots, X_{\ell}(q)\right\}$. The distribution $X$ is said to be involutive if it is closed under operation of Lie bracket, i.e., if for all vector fields $X$ and $Y$ taking values in $X$, the vector field $[X, Y]$ also takes value in $X$. The involutive closure of the distribution $X$ is the smallest involutive distribution containing $X$, and is denoted $\overline{\operatorname{Lie}}\{X\}$.

We define the symmetric product of two vector fields $X$ and $Y$ as the vector field

$$
\langle X: Y\rangle=\langle Y: X\rangle=\nabla_{X} Y+\nabla_{Y} X,
$$

or equivalently

$$
\langle X: Y\rangle^{k}=\frac{\partial Y^{k}}{\partial q^{j}} X^{j}+\frac{\partial X^{k}}{\partial q^{j}} Y^{j}+\Gamma_{i j}^{k}\left(Y^{j} X^{i}+Y^{i} X^{j}\right) .
$$

A distribution $X$ is said to be geodesically invariant if it is closed under operation of symmetric product, i.e., if for all vector fields $X$ and $Y$ taking values in $X$, the vector field $\langle X: Y\rangle$ also takes value in $X$. The symmetric closure of the distribution $X$ is the smallest geodesically invariant distribution containing $\mathcal{X}$, and is denoted $\overline{\operatorname{Sym}}\{X\}$.

\section{Analysis of Kinematic Reduc- tions}

In this section we relate (i) controlled trajectories for the (second-order) controlled Euler-Lagrange equation (1) to (ii) controlled trajectories for driftless control systems on $Q$. The purpose is to establish relationships between the given simple mechanical control system and an appropriate low-complexity kinematic representation.

Assumption 3.1. For the remainder of this section, we restrict our attention to simple mechanical control systems subject to no potential energy and to no dissipative forces, i.e., we set $V=0$, and $F_{\mathrm{diss}}=0$.

Let us start by establishing some nomenclature. We refer to second-order differential equations on $Q$ of the form (1) as dynamic models of mechanical systems; in dynamic models the control inputs are accelerations. In contrast to this, we refer to first-order differential equations on $Q$ as kinematic models of mechanical systems; in kinematic models the control inputs are velocity variables. Let $\mathcal{V}=\left\{V_{1}, \ldots, V_{\ell}\right\}$ be a family of vector fields linearly independent at each $q \in \mathrm{Q}$. For curves $\gamma:[0, T] \rightarrow \mathrm{Q}$ and $w:[0, T] \rightarrow \mathbb{R}^{\ell}$, consider the differential equation

$$
\gamma^{\prime}(t)=\sum_{b=1}^{\ell} V_{b}(\gamma(t)) w_{b}(t) .
$$

We shall assume that the control inputs to kinematic systems take values in the set of absolutely continuous functions $\mathcal{U}_{\text {kin }}^{\ell}$. We shall say that the collection of vector fields $\mathcal{V}$ is a kinematic model or that it induces a kinematic model.

\subsection{Kinematic reductions and decou- pling vector fields}

The kinematic model induced by $\mathcal{V}=\left\{V_{1}, \ldots, V_{\ell}\right\}$ is said to be a kinematic reduction of the dynamic model (1) if, for any control input $w \in \mathcal{U}_{\mathrm{kin}}^{\ell}$ and corresponding controlled trajectory $(\gamma, w)$ for equation (5), there exists a control input $u \in \mathcal{U}_{\mathrm{dyn}}^{m}$ such that $(\gamma, u)$ is a controlled trajectory for the dynamic model (1). In other words, for any curve $\gamma: I \rightarrow \mathrm{Q}$ solving the equation (5) with $w \in$ $\mathcal{U}_{\text {kin }}^{\ell}$, there exists a control $u \in \mathcal{U}_{\mathrm{dyn}}^{m}$ such that $(\gamma, u)$ is a controlled trajectory for the dynamic model (1). Roughly speaking, the curve $\gamma: I \rightarrow Q$ solving (5) can be lifted to a solution to the second-order system (1).

The rank of a kinematic reduction is the rank of the distribution generated by the vector fields $\mathcal{V}$. Rank-one kinematic reductions are particularly interesting. We shall call a vector field $V_{0}$ decoupling if the rank-one kinematic system induced by $\mathcal{V}=\left\{V_{0}\right\}$ is a kinematic reduction. Hence, the second-order control system (1) can be steered along any time-scaled integral curve of a decoupling vector field. For a dynamic control system with a rank- $m$ input distribution, there are at most $m$ rank-one kinematic reductions linearly independent at each $q \in \mathrm{Q}$.

The following theorem characterizes kinematic reductions in terms of the affine connection and the input distribution of the given dynamic model.

Theorem 3.2. (Bullo and Lewis [11]). A kinematic model induced by $\left\{V_{1}, \ldots, V_{\ell}\right\}$ is a kinematic reduction of the simple mechanical control system $\left(Q, \mathbb{G}, V=0, F_{\mathrm{diss}}=\right.$ $0, \mathcal{D}, \mathcal{F})$ if and only if the distribution generated by $V_{i}$ and $\left\langle V_{j}: V_{k}\right\rangle$, for $i, j, k \in\{1, \ldots, \ell\}$ is a subdistribution of the input distribution $y$.

\subsection{Maximally reducible mechanical sys- tems}

We are here interested in characterizing when is a mechanical system kinematic? That is, we are interested in 
when the largest possible kinematic reduction will be attained. By Theorem 3.2, any kinematic reduction must be contained in the distribution $y$, so one can do no better than have the collection of vector fields $\mathcal{Y}$ itself as a kinematic reduction. Formally, we say that the dynamic model (1) is maximally reducible to the kinematic system induced by $\mathcal{V}$ if, $\mathcal{V}$ is a kinematic reduction of (1) and if, for any control input $u \in \mathcal{U}_{\mathrm{dyn}}^{m}$, initial condition $\gamma^{\prime}(0) \in \mathcal{V}$, and corresponding controlled trajectory $(\gamma, u)$ for equation (1), there exists a control input $w \in \mathcal{U}_{\text {kin }}^{\ell}$ such that $(\gamma, w)$ is a controlled trajectory for the kinematic system (5) induced by $\mathcal{V}$. A dynamic system (1) is maximally reducible to a kinematic system if there exists one such collection of vector fields $\mathcal{V}$.

The following theorem characterizes dynamic systems which are maximally reducible.

Theorem 3.3. (Lewis [31]). A simple mechanical control system $\left(Q, \mathbb{G}, V=0, F_{\text {diss }}=0, \mathcal{D}, \mathcal{F}\right)$ with input distribution $\mathrm{y}$ is maximally reducible to a kinematic system if and only if

(i) the kinematic system is induced by $y$, and

(ii) $y$ is geodesically invariant.

\subsection{Computing decoupling vector fields}

According to Theorem 3.2, any decoupling vector field $V_{0}$ takes values in the input distribution $y$. Therefore, we lose no generality by writing

$$
V_{0}=h^{1} Y_{1}+\cdots+h^{m} Y_{m},
$$

where $h^{1}, \ldots, h^{m}$ are arbitrary scalar functions on $\mathbf{Q}$. Then, the vector field $V_{0}$ is decoupling if and only if, for all $q \in \mathrm{Q}$ and for all $1 \leq c \leq n-m$,

$$
0=\sum_{a=1}^{m} \sum_{b=1}^{m}\left(h^{a} h^{b} \mathbb{G}\left(X_{c},\left\langle Y_{a}: Y_{b}\right\rangle\right)\right)(q),
$$

where $\left\{X_{1}, \ldots, X_{n-m}\right\}$ generates the $\mathbb{G}$-orthogonal complement of $y$. A decoupling vector field is therefore identified by a set of functions $h^{1}, \ldots, h^{m}$ solution to the $n-m$ quadratic equations (6). Although these equations may be difficult to treat analytically, solutions can be found on a case-by-case basis by means of symbolic manipulation software. For example, in any three degreesof-freedom system with two control inputs, only one quadratic equation needs to be solved as a function of two unknown functions. It is sometimes possible to design possible decoupling vector fields by relying on physical intuition about the system's behavior. For example, a set of useful concepts comes from the theory of group actions and symmetries; see Section 6 .

\section{Controllability Analysis}

\subsection{Controllable kinematic systems}

We start by defining accessibility and controllability for general kinematic systems. Here we let $\mathcal{V}=\left\{V_{1}, \ldots, V_{\ell}\right\}$ be vector fields on $Q$ giving rise to the driftless nonlinear control system (5). For $q_{0} \in \mathrm{Q}$, we let

$\mathcal{R}^{\mathcal{V}}\left(q_{0}, T\right)=\{\gamma(T) \mid(\gamma, u)$ is a controlled

trajectory for (5) defined on $[0, T]$ with $\left.\gamma(0)=q_{0}\right\}$,

and $\mathcal{R}^{\mathcal{V}}\left(q_{0}, \leq T\right)=\bigcup_{t \in[0, T]} \mathcal{R}^{\mathcal{V}}\left(q_{0}, t\right)$

Definition 4.1. The system (5) is

(i) locally accessible from $q_{0}$ if there exists $T>0$ so that $\operatorname{int}\left(\mathcal{R}^{\mathcal{V}}\left(q_{0}, \leq t\right)\right) \neq \emptyset$ for $t \in(0, T]$, is

(ii) small-time locally controllable (STLC) from $q_{0}$ if there exists $T>0$ so that $q_{0} \in \operatorname{int}\left(\mathcal{R}^{\mathcal{V}}\left(q_{0}, \leq t\right)\right)$ for $t \in(0, T]$, and is

(iii) controllable if for every $q_{1}, q_{2} \in Q$ there exists a controlled trajectory $(\gamma, u)$ defined on $[0, T]$ for some $T>0$ with the property that $\gamma(0)=q_{1}$ and $\gamma(T)=$ $q_{2}$.
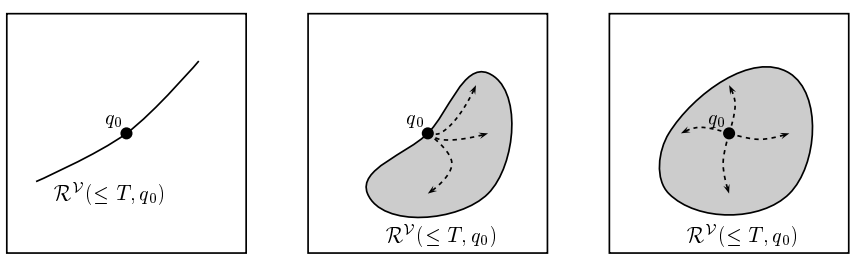

Figure 2: The left figure illustrates a system that is not locally accessible: the reachable set is lower dimensional than the state space. The central figure illustrates a system that is accessible but for which the reachable set does not contain a neighborhood of the initial configuration. The right figure illustrates an STLC system where the reachable set contains a neighborhood of the initial configuration.

Let us state some well-known results concerning the various types of controllability of (5).

Theorem 4.2. (Sussmann and Jurdjevic [49]). The system (5) is STLC (and therefore accessible) from $q_{0}$ if and only if $\overline{\mathrm{Lie}}\{\mathcal{V}\}_{q_{0}}=T_{q_{0}} Q$. Furthermore, if $Q$ is connected and if $\overline{\operatorname{Lie}}\{\mathcal{V}\}_{q}=T_{q} Q$ for each $q \in Q$, then (5) is controllable. 


\subsection{Kinematically controllable dynamic systems}

A dynamic mechanical system (1) described by $\left(\mathrm{Q}, \mathbb{G}, V=0, F_{\text {diss }}=0, \mathcal{D}, \mathcal{F}\right)$ is kinematically controllable if there exists a sequence of kinematic reductions $\left\{\mathcal{V}_{1}, \ldots, \mathcal{V}_{k}\right\}$ with $\operatorname{rank}\left\{\ell_{1}, \ldots, \ell_{k}\right\}$ with the property that, for every $q_{1}, q_{2} \in \mathrm{Q}$, there are corresponding controlled trajectories

$$
\begin{array}{r}
\left\{\left(\gamma_{i}, w_{i}\right) \mid \gamma_{i}:\left[T_{i-1}, T_{i}\right] \rightarrow \mathrm{Q}, w_{i}:\left[T_{i-1}, T_{i}\right] \rightarrow \mathbb{R}^{\ell_{i}},\right. \\
i \in\{1, \ldots, k\}\}
\end{array}
$$

with $\gamma_{1}\left(T_{0}\right)=q_{1}, \gamma_{k}\left(T_{k}\right)=q_{2}$, and $\gamma_{i}\left(T_{i}\right)=\gamma_{i+1}\left(T_{i}\right)$ for all $i \in\{1, \ldots, k-1\}$. In other words, any $q_{2} \in \mathrm{Q}$ is reachable from any $q_{1} \in \mathrm{Q}$ by concatenating motions on $Q$ corresponding to kinematic reductions of (1). The dynamic system (1) is locally kinematically controllable from $q_{0}$ if, for any neighborhood of $q_{0}$ on $\mathrm{Q}$, the set of reachable configurations by trajectories remaining in the neighborhood and following motions of its kinematic reductions contains $q_{0}$ in its interior.

Theorem 4.3. (Bullo and Lynch [13]). A simple mechanical control system $\left(Q, \mathbb{G}, V=0, F_{\text {diss }}=0, \mathcal{D}, \mathcal{F}\right)$ is locally kinematically controllable if and only if it possesses a collection of decoupling vector fields (i.e., rankone kinematic reductions) whose involutive closure has maximal rank everywhere in $Q$.

\subsection{Controllable dynamic systems}

We consider again a dynamic model (1) derived from $\left(\mathrm{Q}, \mathbb{G}, V=0, F_{\text {diss }}=0, \mathcal{D}, \mathcal{F}\right)$. For $q_{0} \in \mathrm{Q}$ we denote

$$
\begin{aligned}
& \mathcal{R}_{T Q}\left(q_{0}, T\right)=\left\{\gamma^{\prime}(T) \mid(\gamma, u)\right. \text { is a controlled } \\
& \left.\quad \text { trajectory of }(1) \text { on }[0, T] \text { satisfying } \gamma^{\prime}(0)=0_{q_{0}}\right\} .
\end{aligned}
$$

Here $0_{q_{0}} \in T_{q_{0}} \mathrm{Q}$ is the zero vector. (Also recall that the tangent vector $\gamma^{\prime}(T)$ uniquely identifies the base point $\gamma(T)$.) We also define $\mathcal{R}_{T Q}\left(q_{0}, \leq T\right)=$ $\bigcup_{t \in[0, T]} \mathcal{R}_{T Q}\left(q_{0}, t\right)$. With these notions of reachable sets, we have the following definitions of controllability.

Definition 4.4. Consider a dynamic mechanical system (1) described by $\left(Q, \mathbb{G}, V=0, F_{\mathrm{diss}}=0, \mathcal{D}, \mathcal{F}\right)$ and let $q_{0} \in Q$. Suppose that the controls for (1) are restricted to take their values in a compact set of $\mathbb{R}^{m}$ which contains 0 in the interior of its convex hull. The system (1) is

(i) locally accessible from $q_{0}$ if there exists $T>0$ so that $\operatorname{int}\left(\mathcal{R}_{T Q}\left(q_{0}, \leq t\right)\right) \neq \emptyset$ for $t \in(0, T]$, and is

(ii) small-time locally controllable (STLC) from $q_{0}$ if there exists $T>0$ so that $0_{q_{0}} \in \operatorname{int}\left(\mathcal{R}_{T Q}\left(q_{0}, \leq t\right)\right)$ for all $t \in(0, T]$.
Before proceeding, we need some notation concerning iterated symmetric products in the vector fields $\left\{Y_{1}, \ldots, Y_{m}\right\}$. Such a symmetric product is bad if it contains an even number of each of the vector fields $Y_{1}, \ldots, Y_{m}$, and otherwise is good. Thus, for example, $\left\langle\left\langle Y_{a}: Y_{b}\right\rangle:\left\langle Y_{a}: Y_{b}\right\rangle\right\rangle$ is bad for all $a, b \in\{1, \ldots, m\}$ and $\left\langle Y_{a}:\left\langle Y_{b}: Y_{c}\right\rangle\right\rangle$ and $\left\langle Y_{a}:\left\langle Y_{b}: Y_{b}\right\rangle\right\rangle$ are good for any $a, b, c \in\{1, \ldots, m\}$. The degree of a symmetric product is the total number of input vector fields comprising the symmetric product. For example, $\left\langle\left\langle Y_{a}: Y_{b}\right\rangle:\left\langle Y_{a}: Y_{b}\right\rangle\right\rangle$ has degree 4 and $\left\langle Y_{a}:\left\langle Y_{b}: Y_{c}\right\rangle\right\rangle$ has degree 3. If $P$ is a symmetric product in the vector fields $\left\{Y_{1}, \ldots, Y_{m}\right\}$ and if $\sigma \in S_{m}$ is an element of the permutation group on $\{1, \ldots, m\}, \sigma(P)$ denotes the symmetric product obtained by replacing each occurrence of $Y_{a}$ with $Y_{\sigma(a)}$.

We now state the main result concerning controllability in state space of dynamic mechanical systems.

Theorem 4.5. (Lewis and Murray [33]). A simple mechanical control system $\left(Q, \mathbb{G}, V=0, F_{\mathrm{diss}}=0, \mathcal{D}, \mathcal{F}\right)$ is

(i) locally accessible from $q_{0} \in Q$ if and only if $\overline{\operatorname{Sym}}\{y\}_{q_{0}}=T_{q_{0}} Q$, and is

(ii) $S T L C$ from $q_{0} \in Q$ if $\overline{\operatorname{Sym}}\{y\}_{q_{0}}=T_{q_{0}} Q$ and if for every bad symmetric product $P$ we have

$$
\sum_{\sigma \in S_{m}} \sigma(P)\left(q_{0}\right) \in \operatorname{span}_{\mathbb{R}}\left\{P_{1}\left(q_{0}\right), \ldots, P_{k}\left(q_{0}\right)\right\},
$$

where $P_{1}, \ldots, P_{k}$ are good symmetric products of degree less than $P$.

\subsection{Configuration controllable dynamic systems}

The preceding discussion concerned the set of reachable states for a dynamic mechanical system. Let us now restrict to descriptions of the set of reachable configurations. For $q_{0} \in \mathrm{Q}$ we denote

$$
\begin{aligned}
& \mathcal{R}_{\mathrm{Q}}\left(q_{0}, T\right)=\{\gamma(T) \mid(\gamma, u) \text { is a controlled } \\
& \left.\quad \text { trajectory of }(1) \text { on }[0, T] \text { satisfying } \gamma^{\prime}(0)=0_{q_{0}}\right\} .
\end{aligned}
$$

We also define $\mathcal{R}_{\mathbf{Q}}\left(q_{0}, \leq T\right)=\bigcup_{t \in[0, T]} \mathcal{R}_{\mathbf{Q}}\left(q_{0}, t\right)$. This gives the following notions of controllability relative to configurations.

Definition 4.6. Consider a dynamic mechanical system (1) described by $\left(Q, \mathbb{G}, V=0, F_{\mathrm{diss}}=0, \mathcal{D}, \mathcal{F}\right)$ and let $q_{0} \in Q$. The dynamic mechanical system (1) is

(i) locally configuration accessible from $q_{0}$ if there exists $T>0$ so that $\operatorname{int}\left(\mathcal{R}_{Q}\left(q_{0}, \leq t\right)\right) \neq \emptyset$ for all $t \in(0, T]$, and is 
(ii) small-time locally configuration controllable (STLCC) from $q_{0}$ if there exists $T>0$ so that $q_{0} \in \operatorname{int}\left(\mathcal{R}_{Q}\left(q_{0}, \leq t\right)\right)$ for all $t \in(0, T]$ with the controls restricted to take their values in a compact subset of $\mathbb{R}^{m}$ that contains the origin in its convex hull.

Theorem 4.7. (Lewis and Murray [33]). Consider a dynamic mechanical system (1) described by $(Q, \mathbb{G}, V=$ $\left.0, F_{\mathrm{diss}}=0, \mathcal{D}, \mathcal{F}\right)$ and let $q_{0} \in Q$. The dynamic mechanical system (1) is

(i) locally configuration accessible from $q_{0}$ if and only if $\overline{\operatorname{Lie}}\{\overline{\operatorname{Sym}}\{y\}\}_{q_{0}}=T_{q_{0}} Q$, and is

(ii) STLCC from $q_{0}$ if $\overline{\operatorname{Lie}}\{\overline{\operatorname{Sym}}\{y\}\}_{q_{0}}=T_{q_{0}} Q$ and if for every bad symmetric product $P$ we have

$$
\sum_{\sigma \in S_{m}} \sigma(P)\left(q_{0}\right) \in \operatorname{span}_{\mathbb{R}}\left\{P_{1}\left(q_{0}\right), \ldots, P_{k}\left(q_{0}\right)\right\},
$$

where $P_{1}, \ldots, P_{k}$ are good symmetric products of degree less than $P$.

\subsection{Controllability inferences and exam- ples}

We summarize the relationships between the various controllability concepts introduced up until here. In particular, Figure 3 illustrates the relationships between small-time locally controllable (STLC), small-time locally configuration controllable (STLCC), locally kinematically controllable (LKC), and maximally reducible, locally kinematically controllable (MR-LKC) systems. All implications in the figure are clear from the theoretical treatment. Without further assumptions on the

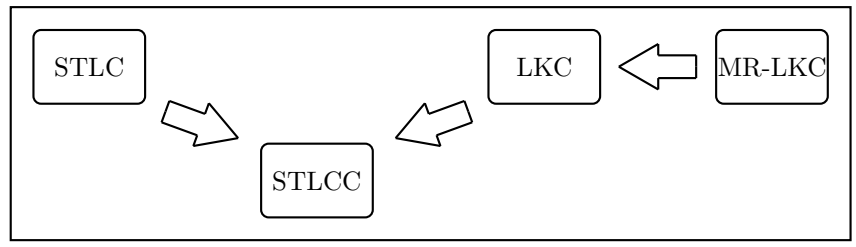

Figure 3: Inference between controllability notions for mechanical control systems

dimension of the configuration space and on the rank of the input distribution, no further implications can be added to Figure 3.

Finally, we consider a number of instructive examples and present a summary description of their kinematic reductions and of their controllability properties. The catalog is presented in Table 4.5 on page 8 . The various statements in the table are obtained in $[10,13,14,15$, $30,31,35]$.

\section{Averaging Analysis}

In this section we characterize the controlled trajectories for a simple mechanical system subject to oscillatory input forces, i.e., input forces of large amplitude and high frequency. To perform the averaging analysis, we start by introducing certain useful iterated integrals of the input functions.

For $a \in\{1, \ldots, m\}$, let $u_{a}: \overline{\mathbb{R}}_{+} \times \overline{\mathbb{R}}_{+} \rightarrow \mathbb{R}$ be bounded functions, $T$-periodic and zero-mean in their first argument, continuously differentiable in their second argument. These functions are the control inputs for the simple mechanical system and it is convenient to let them depend on time in two different ways. For $a, b \in\{1, \ldots, m\}$, let

$$
\begin{aligned}
U_{(a)}(\tau, t) & =\int_{0}^{t} u_{a}(\tau, s) d s \\
U_{(a, b)}(\tau, t) & =\int_{0}^{t} u_{b}\left(\tau, s_{2}\right) \int_{0}^{s_{2}} u_{a}\left(\tau, s_{1}\right) d s_{1} d s_{2},
\end{aligned}
$$

and let $\bar{U}_{(a)}, \bar{U}_{(a, b)}: \overline{\mathbb{R}}_{+} \rightarrow \mathbb{R}$ be their averages over $[0, T]$ with respect to their first argument. Let $\Lambda$ be a curve of $n \times n$ symmetric matrices given by

$$
\Lambda_{a b}(t)=\frac{1}{2}\left(\bar{U}_{(a, b)}(t)+\bar{U}_{(b, a)}(t)-\bar{U}_{(a)}(t) \bar{U}_{(b)}(t)\right) .
$$

One can show that, for all $t \in \overline{\mathbb{R}}_{+}, \Lambda_{a b}(t)$ is positive semi-definite. Furthermore, for any $t_{0} \in \overline{\mathbb{R}}_{+}$such that all $\tau \mapsto u_{1}\left(\tau, t_{0}\right), \ldots, u_{m}\left(\tau, t_{0}\right)$ are non zero, $\Lambda_{a b}\left(t_{0}\right)$ is positive definite.

For $f, g: \mathbb{R} \rightarrow \mathbb{R}^{n}$, we write $f(\epsilon) \approx g(\epsilon)$ as $\epsilon \rightarrow 0$ if $\|f(\epsilon)-g(\epsilon)\| \rightarrow 0$ in the limit as $\epsilon \rightarrow 0$.

Theorem 5.1. (Martinez et al. [38]). Consider the simple mechanical control system $\left(Q, \mathbb{G}, V, F_{\text {diss }}, \mathcal{D}, \mathcal{F}\right)$ with governing equation in $\gamma: I \rightarrow Q$

$$
\begin{array}{r}
\nabla_{\gamma^{\prime}(t)} \gamma^{\prime}(t)=-P\left(\operatorname{grad} V(\gamma(t))+\mathbb{G}^{-1}\left(F_{\text {diss }}\left(\gamma^{\prime}(t)\right)\right)\right) \\
+\sum_{a=1}^{m} \frac{1}{\epsilon} u_{a}\left(\frac{t}{\epsilon}, t\right) Y_{a}(\gamma(t)),
\end{array}
$$

for $\epsilon>0$ and with initial condition $\gamma^{\prime}(0) \in \mathcal{D}$. Consider the initial value problem in $\xi: I \rightarrow Q$

$$
\begin{aligned}
& \nabla_{\xi^{\prime}(t)} \xi^{\prime}(t)=-P\left(\operatorname{grad} V(\xi(t))+\mathbb{G}^{-1}\left(F_{\text {diss }}\left(\xi^{\prime}(t)\right)\right)\right) \\
& -\sum_{a, b=1}^{m} \Lambda_{a b}(t)\left\langle Y_{a}: Y_{b}\right\rangle(\xi(t)) \text {, }
\end{aligned}
$$

with $\xi^{\prime}(0)=\gamma^{\prime}(0)+\sum_{a=1}^{m} \bar{U}_{(a)}(0) Y_{a}(\gamma(0))$. Then, for all 
Table 1: Catalog of low-dimensional simple mechanical control systems

\begin{tabular}{|c|c|c|}
\hline System & Picture & Reducibility \& Controllability \\
\hline $\begin{array}{l}\text { planar } 2 \mathrm{R} \text { robot } \\
\text { single torque at either joint: } \\
(1,0),(0,1) \\
n=2, m=1\end{array}$ & & $\begin{array}{l}(1,0) \text { : no reductions, accessible } \\
(0,1) \text { : decoupling v.f., maximally re- } \\
\text { ducible, not accessible or STLCC }\end{array}$ \\
\hline $\begin{array}{l}\text { roller racer } \\
\text { single torque at joint } \\
n=4, m=1\end{array}$ & & $\begin{array}{l}\text { no kinematic reductions, accessible, not } \\
\text { STLCC }\end{array}$ \\
\hline $\begin{array}{l}\text { planar body with single force } \\
\text { or torque } \\
n=3, m=1\end{array}$ & & decoupling v.f., reducible, not accessible \\
\hline $\begin{array}{l}\text { planar body with single gen- } \\
\text { eralized force } \\
n=3, m=1\end{array}$ & & $\begin{array}{l}\text { no kinematic reductions, accessible, not } \\
\text { STLCC }\end{array}$ \\
\hline $\begin{array}{l}\text { planar body with two forces } \\
n=3, m=2\end{array}$ & & two decoupling v.f., LKC, STLC \\
\hline $\begin{array}{l}\text { robotic leg } \\
n=3, m=2\end{array}$ & & $\begin{array}{l}\text { two decoupling v.f., maximally reducible } \\
\text { and LKC }\end{array}$ \\
\hline $\begin{array}{l}\text { planar } 3 \mathrm{R} \text { robot, two } \\
\text { torques: } \\
(0,1,1),(1,0,1),(1,1,0) \\
n=3, m=2\end{array}$ & & $\begin{array}{l}(1,0,1) \text { and }(1,1,0) \text { : two decoupling v.f., } \\
\text { LKC and STLC } \\
(0,1,1) \text { : two decoupling v.f., maximally } \\
\text { reducible and LKC }\end{array}$ \\
\hline $\begin{array}{l}\text { rolling penny } \\
n=4, m=2\end{array}$ & & maximally reducible and LKC \\
\hline $\begin{array}{l}\text { snakeboard } \\
n=5, m=2\end{array}$ & & two decoupling v.f., LKC, STLCC \\
\hline $\begin{array}{l}3 \mathrm{D} \text { vehicle with } 3 \text { generalized } \\
\text { forces } \\
n=6, m=3\end{array}$ & & three decoupling v.f., LKC, STLC \\
\hline
\end{tabular}


time $t$ in a finite interval $[0, \bar{t}]$

$$
\begin{aligned}
\gamma(t) & \approx \xi(t), \\
\gamma^{\prime}(t) & \approx \xi^{\prime}(t)+\sum_{a=1}^{m}\left(U_{(a)}(t / \epsilon, t)-\bar{U}_{(a)}(t)\right) Y_{a}(\xi(t)),
\end{aligned}
$$

as $\epsilon \rightarrow 0$.

In simple words, the theorem states that, with some specified level of accuracy, the averaged evolution of a simple mechanical control system subject to oscillatory inputs is equal to that of a simple mechanical system subject to an "appropriate" force. This "appropriate" force is a linear combination of symmetric products of the control vector fields with coefficients computed via appropriate iterated integrals of the input functions.

\section{Systems with potential control forces and the av- eraged potential}

Here we consider simple mechanical systems with potential control forces. We will show how their averaged behavior can be described as that of a simple mechanical system with, as potential energy, the so-called averaged potential.

Let $\left(\mathrm{Q}, \mathbb{G}, V, F_{\text {diss }}, \mathcal{D}=T \mathrm{Q}, \mathcal{F}=\left\{\mathrm{d} \varphi^{1}, \ldots, \mathrm{d} \varphi^{m}\right\}\right)$ be a simple mechanical control system subject to dissipation and with potential control forces. (Note the absence of a constraint distribution.) The governing equations of motion take the specific form

$$
\begin{array}{r}
\mathbb{G}_{\gamma^{\prime}} \gamma^{\prime}=-\operatorname{grad} V(\gamma)+\mathbb{G}^{-1}\left(F_{\text {diss }}\left(\gamma^{\prime}\right)\right) \\
+\sum_{a=1}^{m} u^{a}(t) \operatorname{grad}\left(\varphi^{a}\right)(\gamma) .
\end{array}
$$

The following result is useful to analyze this class of systems.

Lemma 5.2. (Crouch [19]). Let $\langle\cdot: \cdot\rangle$ be the symmetric product induced by the Levi-Civita connection of $(Q, \mathbb{G})$. For any smooth $\varphi^{1}, \varphi^{2}: Q \rightarrow \mathbb{R}$, the symmetric product $\left\langle\operatorname{grad} \varphi^{1}: \operatorname{grad} \varphi^{2}\right\rangle$ is a gradient vector field. Additionally, if one defines

$$
\left\langle\varphi^{1}: \varphi^{2}\right\rangle=\mathbb{G}\left(\operatorname{grad} \varphi^{1}, \operatorname{grad} \varphi^{2}\right),
$$

then

$$
\left\langle\operatorname{grad} \varphi^{1}: \operatorname{grad} \varphi^{2}\right\rangle=\operatorname{grad}\left\langle\varphi^{1}: \varphi^{2}\right\rangle .
$$

In other words, this lemma stats that the set of gradient vector fields is closed under the operation of symmetric product. Next, we apply Theorem 5.1 to simple mechanical control systems with potential forces, and simplify the averaged systems exploiting the result in Lemma 5.2.
Theorem 5.3. (Bullo [9]). Consider the simple mechanical control system subject to dissipation and with potential control forces $\left(Q, \mathbb{G}, V, F_{\text {diss }}, \mathcal{D}=T Q, \mathcal{F}=\right.$ $\left.\left\{\mathrm{d} \varphi^{1}, \ldots, \mathrm{d} \varphi^{m}\right\}\right)$. For $i \in\{1, \ldots, m\}$, let the functions $u_{i}: \overline{\mathbb{R}}_{+} \rightarrow \mathbb{R}$ be bounded, T-periodic, and zero-mean. Then, the averaged system satisfies the (unforced) equations of motion

$$
{ }^{\mathbb{G}} \nabla_{\xi^{\prime}} \xi^{\prime}=-\operatorname{grad} V_{\text {averaged }}(\xi)+\mathbb{G}^{-1}\left(F_{\text {diss }}\left(\xi^{\prime}\right)\right) .
$$

where the averaged potential $V_{\text {averaged }}: Q \rightarrow \mathbb{R}$ is

$$
V_{\text {averaged }}=V+\sum_{a, b=1}^{m} \Lambda_{a b}\left\langle\varphi^{a}: \varphi^{b}\right\rangle .
$$

Here $\Lambda$ is defined as above; however, the control inputs depend only on one time scale and therefore $\Lambda$ is constant.

\section{Trajectory Design via Inverse Kinematics}

In this section we consider the problem of designing a controlled trajectory for a simple mechanical control system $\left(\mathrm{Q}, \mathbb{G}, V=0, F_{\text {diss }}=0, \mathcal{D}, \mathcal{F}\right)$ connecting two arbitrary initial and target configurations $q_{\text {initial }}$ and $q_{\text {target }}$ in $\mathrm{Q}$. We shall tackle this problem by introducing the notion of infinitesimal generators and making an appropriate assumption.

Let $\mathrm{G}$ be a Lie group, $\mathfrak{g}$ be its Lie algebra and let exp: $\mathfrak{g} \rightarrow \mathrm{G}$ be the corresponding exponential map. Given a configuration manifold $\mathrm{Q}$, a right group action is a smooth map $\Phi: \mathrm{Q} \times \mathrm{G} \rightarrow \mathrm{Q}$, such that $\Phi(q, e)=q$ for all $q \in \mathrm{Q}$, where $e$ denotes the identity element in $\mathrm{G}$, and $\Phi(\Phi(q, g), h)=\Phi(q, g h)$ for all $g, h \in \mathrm{G}, q \in \mathrm{Q}$. For $\xi \in \mathfrak{g}$, the infinitesimal generator $V_{\xi}$ is the vector field on $Q$ defined by

$$
V_{\xi}(q)=\left.\frac{\mathrm{d}}{\mathrm{d} t}\right|_{t=0} \Phi(q, \exp (t \xi)) .
$$

Note that the flow of infinitesimal generators can be computed in closed form by means of the exponential map and group action.

Group actions play an interesting role when paired with kinematic reductions of simple mechanical control systems. Numerous examples from Table 4.5 enjoy the following property: the decoupling vector fields turn out to be infinitesimal generators of some group action. Indeed, oftentimes decoupling vector fields correspond to infinitesimal rotations and translations (measured with respect to the body-frame). In these cases, the group $\mathrm{G}$ is a subgroup of $\operatorname{SE}(n)$ and the group action is a rigid 
displacement of some components of the mechanical device.

Assumption 6.1. In what follows, we assume that the dynamic mechanical control system $\left(Q, \mathbb{G}, V=0, F_{\text {diss }}=\right.$ $0, \mathcal{D}, \mathcal{F})$ is kinematically controllable and admits a set of decoupling vector fields that are infinitesimal generators of an appropriate group action $\Phi: Q \times \mathrm{G} \rightarrow Q$. Furthermore, we assume the motion planning problem satisfies

$$
\Phi\left(q_{\text {initial }}, g_{\text {desired }}\right)=q_{\text {target }}
$$

for some appropriate $g_{\mathrm{desired}} \in \mathrm{G}$.

Note that these assumptions do not imply that the mechanical system is invariant under the group action.

In summary, we are given a family of left-invariant vector fields $\left\{V_{1}, \ldots, V_{m}\right\}$ on $\mathrm{G}$ and we consider the associated driftless control system

$$
\dot{g}(t)=\sum_{i=1}^{m} V_{i}(g(t)) w_{i}(t)
$$

where $t \mapsto g(t) \in \mathrm{G}$ and where $t \mapsto\left(w_{1}, \ldots, w_{m}\right) \in$ $\{( \pm 1,0, \ldots, 0),(0, \pm 1,0, \ldots, 0), \ldots,(0, \ldots, 0, \pm 1)\} . \quad$ (As common in matters of Lie group theory, we identify leftinvariant vector fields with their value at the identity.)

The design objective is to compute feasible motion plans for the control system (7) by concatenating a finite number of flows along the input vector fields. Trajectory design is reduced to the problem of selecting a finite-length combination of $k$ maneuvers $\left\{\left(i_{1}, \ldots, i_{k}\right) \mid i_{j} \in\{1, \ldots, m\}\right\}$ and computing appropriate coasting times $\left\{t_{1}, \ldots, t_{k}\right\}$ that steer the system from the identity in the group to any target configuration $g_{\text {desired }} \in$ G. In mathematical terms, we need to solve

$$
\begin{gathered}
g_{\text {desired }}=\exp \left(t_{1} V_{i_{1}}\right) \cdots \exp \left(t_{k} V_{i_{k}}\right) \\
k \in \mathbb{N}, t_{1}, \ldots, t_{k} \in \mathbb{R}, i_{1}, \ldots, i_{k} \in\{1, \ldots, m\} .
\end{gathered}
$$

Although no general methodology is available to treat analytically problems of the form (8), it is possible to develop a catalog of solutions for relevant example systems. In what follows we provide planning algorithms for any controllable system evolving on $\mathrm{SO}(3)$, and we refer to [39] for a comprehensive catalog.

\section{Solutions for kinematic systems on $\mathrm{SO}(3)$}

Let $\left\{\widehat{e}_{x}, \widehat{e}_{y}, \widehat{e}_{z}\right\}$ be the basis of $\mathfrak{s o}(3)$ :

$\widehat{e}_{x}=\left[\begin{array}{ccc}0 & 0 & 0 \\ 0 & 0 & -1 \\ 0 & 1 & 0\end{array}\right], \widehat{e}_{y}=\left[\begin{array}{ccc}0 & 0 & 1 \\ 0 & 0 & 0 \\ -1 & 0 & 0\end{array}\right], \widehat{e}_{z}=\left[\begin{array}{ccc}0 & -1 & 0 \\ 1 & 0 & 0 \\ 0 & 0 & 0\end{array}\right]$
Here we make use of the notation $\widehat{V}=a \widehat{e}_{x}+b \widehat{e}_{y}+$ $c \widehat{e}_{z} \equiv(\widehat{a, b, c})$ based on the Lie algebra isomorphism $\widehat{\cdot}:\left(\mathbb{R}^{3}, \times\right) \rightarrow(\mathfrak{s o}(3),[\cdot, \cdot])$. An expression of the exponential exp: $\mathfrak{s o}(3) \rightarrow \mathrm{SO}(3)$ is given in terms of Rodrigues' Formula:

$$
\exp (\widehat{\eta})=I_{3}+\frac{\sin \|\eta\|}{\|\eta\|} \widehat{\eta}+\frac{1-\cos \|\eta\|}{\|\eta\|^{2}} \widehat{\eta}^{2} .
$$

The commutator relations are $\left[\widehat{e}_{x}, \widehat{e}_{z}\right]=-\widehat{e}_{y},\left[\widehat{e}_{y}, \widehat{e}_{z}\right]=$ $\widehat{e}_{x}$ and $\left[\widehat{e}_{x}, \widehat{e}_{y}\right]=\widehat{e}_{z}$.

Consider two left-invariant vector fields $V_{1}=$ $\left(a_{1}, b_{1}, c_{1}\right)$ and $V_{2}=\left(a_{2}, b_{2}, c_{2}\right)$ in $\mathfrak{s o}(3)$. Their Lie closure is full rank if and only if $c_{1} a_{2}-a_{1} c_{2} \neq 0$ or $b_{1} c_{2}-c_{1} b_{2} \neq 0$ or $b_{1} a_{2}-a_{1} b_{2} \neq 0$. Therefore, without loss of generality, we can assume $V_{1}=e_{z}$ (otherwise we perform a suitable change of coordinates), and $\left\|V_{2}\right\|^{2}=1$. In what follows, we let $V_{2}=(a, b, c)$. Since $e_{z}$ and $V_{2}$ are linearly independent, necessarily $a^{2}+b^{2} \neq 0$ and $c \neq \pm 1$.

Since $\operatorname{dim}(\mathfrak{s o}(3))=3$, at least three maneuvers along the flows of $\left\{V_{1}, V_{2}\right\}$ are required to plan a motion between any two arbitrary desired configurations. Thus, we consider the map $\mathcal{F} \mathcal{K}: \mathbb{R}^{3} \rightarrow \mathrm{SO}(3)$

$$
\mathcal{F} \mathcal{K}\left(t_{1}, t_{2}, t_{3}\right)=\exp \left(t_{1} \widehat{e}_{z}\right) \exp \left(t_{2} \widehat{V}_{2}\right) \exp \left(t_{3} \widehat{e}_{z}\right) .
$$

In the following theorem, we design an inverse kinematic map $\mathcal{I} \mathcal{K}$ that will compute appropriate coasting times. In what follows, atan 2 denotes the inverse tangent map that discerns the appropriate quadrant.

Theorem 6.2. (Martínez et al. [39]). Let $V_{1}=(0,0,1)$ and $V_{2}=(a, b, c)$, with $a^{2}+b^{2} \neq 0$ and $c \neq \pm 1$. Define the neighborhood of the identity in $\mathrm{SO}(3)$

$$
U=\left\{R \in \mathrm{SO}(3) \mid R_{33} \in\left[2 c^{2}-1,1\right]\right\} .
$$

Consider the map $\mathcal{I} \mathcal{K}: U \subset \mathrm{SO}(3) \rightarrow \mathbb{R}^{3}$ whose components are

$$
\begin{aligned}
& \mathcal{I K}_{1}(R)=\operatorname{atan} 2\left(w_{1} R_{13}+w_{2} R_{23},-w_{2} R_{13}+w_{1} R_{23}\right), \\
& \mathcal{I K}_{2}(R)=\operatorname{acos}\left(\frac{R_{33}-c^{2}}{1-c^{2}}\right), \\
& \mathcal{I K}_{3}(R)=\operatorname{atan} 2\left(v_{1} R_{31}+v_{2} R_{32}, v_{2} R_{31}-v_{1} R_{32}\right),
\end{aligned}
$$

where, for $z=\left(1-\cos \left(\mathcal{I} \mathcal{K}_{2}(R)\right), \sin \left(\mathcal{I} \mathcal{K}_{2}(R)\right)\right)^{T}$,

$$
\left[\begin{array}{l}
w_{1} \\
w_{2}
\end{array}\right]=\left[\begin{array}{cc}
a c & b \\
c b & -a
\end{array}\right] z, \quad\left[\begin{array}{l}
v_{1} \\
v_{2}
\end{array}\right]=\left[\begin{array}{cc}
a c & -b \\
c b & a
\end{array}\right] z .
$$

Then, $\mathcal{I K}$ is a local right inverse of $\mathcal{F} \mathcal{K}$, that is, it satisfies $\mathcal{F} \mathcal{K} \circ \mathcal{I K}=\mathrm{id}_{U}: U \rightarrow U$.

We illustrate the performance of the algorithms in Figure 4. 


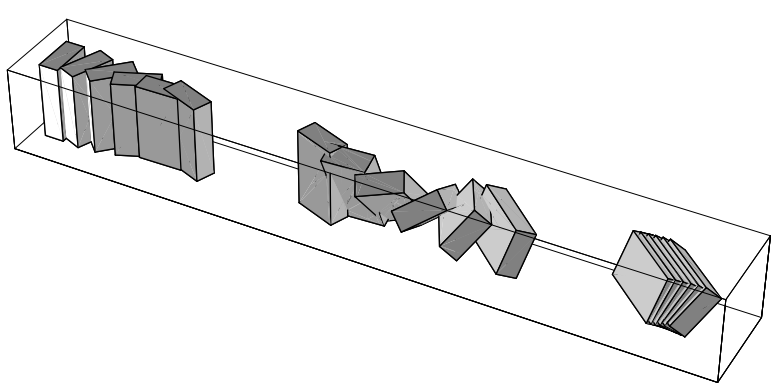

Figure 4: We illustrate the inverse-kinematic planner on $\mathrm{SO}(3)$. The system parameters are $(a, b, c)=(0,1 / \sqrt{2}, 1 / \sqrt{2})$. The target final rotation is $\exp (\pi / 3, \pi / 3,0)$. To render the sequence of three rotations visible, the body is translated along the inertial $x$-axis.

\section{Trajectory Design via Approxi- mate Inversion}

In this section we consider the problem of steering the configuration of the simple mechanical control system $\left(\mathrm{Q}, \mathbb{G}, V, F_{\text {diss }}, \mathcal{D}, \mathcal{F}\right)$ along a pre-specified target trajectory. We do so by means of appropriate oscillatory controls, that is, controls of large amplitude and high frequency as considered in Section 5.

We consider the mechanical control system with constraints $\left(\mathrm{Q}, \mathbb{G}, V, F_{\text {diss }}, \mathcal{D}, \mathcal{F}\right)$ with governing equation in $\gamma: I \rightarrow \mathrm{Q}$

$$
\begin{aligned}
\nabla_{\gamma^{\prime}(t)} \gamma^{\prime}(t)=-P(\operatorname{grad} V(\gamma) & \left.+\mathbb{G}^{-1}\left(F_{\mathrm{diss}}\left(\gamma^{\prime}\right)\right)\right) \\
& +\sum_{a=1}^{m} u_{a} Y_{a}(\gamma(t)),
\end{aligned}
$$

with initial condition $\gamma^{\prime}(0) \in \mathcal{D}$. We state the control objective as follows: given a desired smooth curve $\gamma_{\text {target }}:[0, T] \rightarrow Q$ with initial condition $\gamma_{\text {target }}^{\prime}(0)=$ $\gamma^{\prime}(0)$, find controls $u_{a}: T Q \times[0, T] \rightarrow \mathbb{R}^{m}$ such that the controlled trajectory $\gamma$ approximates $\gamma_{\text {target }}$ up to $O(\epsilon)$ errors.

Assumption 7.1. We assume that $\operatorname{span}_{\mathbb{R}}\left\{Y_{a},\left\langle Y_{b}\right.\right.$ : $\left.\left.Y_{c}\right\rangle \mid a, b, c \in\{1, \ldots, m\}\right\}$ is full rank and that, for all $a \in\{1, \ldots, m\}$, the bad symmetric product $\left\langle Y_{a}: Y_{a}\right\rangle$ takes values in $y$.

This assumption has two immediate consequences. First, there exist functions $z_{\text {target }}^{a}, z_{\text {target }}^{a b}:[0, T] \rightarrow \mathbb{R}$, $a<b$, with

$$
\begin{aligned}
& \nabla_{\gamma_{\text {target }}^{\prime}(t)} \gamma_{\text {target }}^{\prime}(t) \\
& =-P(\operatorname{grad}
\end{aligned}
$$

Second, for $a, b \in\{1, \ldots, m\}$, there exist functions $\alpha_{a, b}: \mathbf{Q} \rightarrow \mathbb{R}$ such that

$$
\left\langle Y_{a}: Y_{a}\right\rangle=\sum_{b=1}^{m} \alpha_{a, b} Y_{b} .
$$

Roughly speaking, we regards the functions $z_{\text {target }}^{a}, z_{\text {target }}^{a b}:[0, T] \rightarrow \mathbb{R}, a<b$, as "virtual inputs" that the control system can only in part realize.

Next, note that there are $N=m(m-1) / 2$ pairs of elements $(a, b)$ in $\{1, \ldots, m\}$, with $a<b$. Let $(a, b) \mapsto$ $\omega(a, b) \in\{1, \ldots, N\}$ be an enumeration of these pairs, and define the scalar functions

$$
\psi_{\omega(a, b)}(t)=\sqrt{2} \omega(a, b) \cos (\omega(a, b) t) .
$$

We are now ready to state the main result of this section on the design of oscillatory controls.

Theorem 7.2. (Martinez et al. [38]). Consider the mechanical control system $\left(Q, \mathbb{G}, V, F_{\text {diss }}, \mathcal{D}, \mathcal{F}\right)$. Let $\gamma_{\text {target }}:[0, T] \rightarrow Q$ be a smooth reference trajectory. Design the feedback and feedforward control laws $u_{a}$ according to $u_{a}=v_{a}(t, q)+(1 / \epsilon) w_{a}(t / \epsilon, t)$ with

$$
\begin{aligned}
v_{a}(t, q) & =z_{\text {target }}^{a}(t) \\
& +\frac{1}{2} \sum_{b=1}^{m} \alpha_{a, b}(q)\left(a-1+\sum_{c=a+1}^{m}\left(z_{\text {target }}^{b c}(t)\right)^{2}\right), \\
w_{a}(\tau, t) & =\sum_{c=a+1}^{m} z_{\text {target }}^{a c}(t) \psi_{\omega(a, c)}(\tau)-\sum_{c=1}^{a-1} \psi_{\omega(c, a)}(\tau) .
\end{aligned}
$$

If $\gamma_{\text {target }}^{\prime}(0)=\gamma^{\prime}(0)$, then the controlled trajectory $\gamma$, solution to equation (10), follows $\gamma_{\text {target }}$ with an error of order $\epsilon$ over the time scale 1.

Roughly speaking, we refer to Theorem 7.2 as an "approximate inversion" of the "averaging procedure" described in Theorem 5.1: given a desired "appropriate force" described by the virtual inputs, we design such oscillatory controls that the average system will be subject to precisely the appropriate force. In other words, 
the simple mechanical system subject to controls $u_{a}$ as designed in the theorem statement satisfies, on average, the differential equation defining the virtual inputs. Note that (1) the virtual inputs $z_{\text {target }}^{a}$ are realized by means of $v_{a}$ terms, (2) the virtual inputs $z_{\text {target }}^{a b}, a<b$, are realized by means of the $w_{a}$ terms via the multiplication with appropriate oscillations, and (3) the second term in the definition of $v_{a}$ is responsible for removing the undesired effects of bad symmetric products during the averaging procedure. A more comprehensive explanation, as well as the proof, of this result is provided in [38]. We illustrate this control design with two example systems.

\section{A second-order nonholonomic integrator}

We consider a dynamical extensions of Brockett's nonholonomic integrator:

$$
\ddot{x}_{1}=u_{1}, \quad \ddot{x}_{2}=u_{2}, \quad \ddot{x}_{3}=u_{1} x_{2}+u_{2} x_{1},
$$

and note that this system fulfills the controllability Assumption 7.1. Following Theorem 7.2, we design control inputs to track a desired trajectory, $t \mapsto$ $\left(x_{1}^{d}(t), x_{2}^{d}(t), x_{3}^{d}(t)\right)$, and obtain

$$
\begin{aligned}
& u_{1}=\ddot{x}_{1}^{d}+\frac{1}{\sqrt{2} \epsilon}\left(\ddot{x}_{3}^{d}-\ddot{x}_{1}^{d} x_{2}^{d}-\ddot{x}_{2}^{d} x_{1}^{d}\right) \cos \left(\frac{t}{\epsilon}\right), \\
& u_{2}=\ddot{x}_{2}^{d}-\frac{\sqrt{2}}{\epsilon} \cos \left(\frac{t}{\epsilon}\right) .
\end{aligned}
$$

An illustration of the performance of these controls is shown in Figure 5.
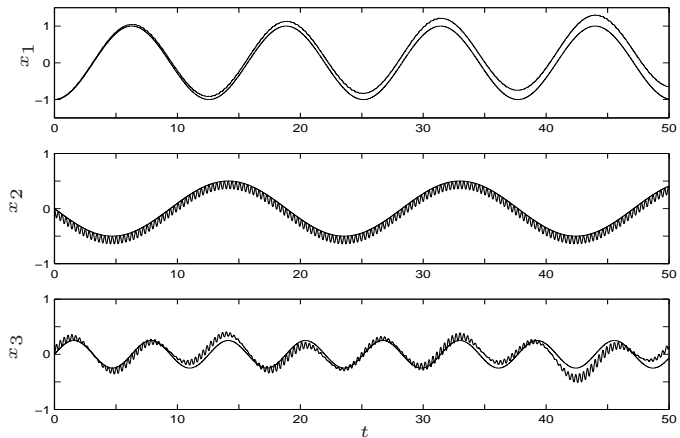

Figure 5: Trajectory design for the second-order nonholonomic integrator $(\epsilon=.05)$

\section{A PVTOL aircraft model}

We consider the model of a simple planar vertical takeoff and landing aircraft model with added viscous damping forces; see Figure 6. We parametrize its con-

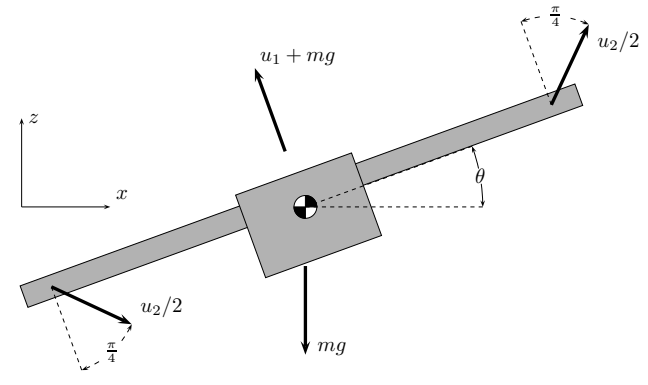

Figure 6: Diagram of the PVTOL aircraft model

figuration and velocity space via the state variables $\left(x, z, \theta, v_{x}, v_{z}, \omega\right)$. We let $x$ and $z$ be the horizontal and vertical displacement of the aircraft, and $\theta$ be its roll angle. The angular velocity is $\omega$ and the linear velocities in the body-fixed $x$ (respectively $z$ ) axis are $v_{x}$ (respectively $\left.v_{z}\right)$. The equations are written as:

$$
\begin{aligned}
\dot{x} & =\cos \theta v_{x}-\sin \theta v_{z}, \\
\dot{z} & =\sin \theta v_{x}+\cos \theta v_{z}, \\
\dot{\theta} & =\omega, \\
\dot{v}_{x} & =\left(-k_{1} / m\right) v_{x}-g \sin \theta+v_{z} \omega+(1 / m) u_{2}, \\
\dot{v}_{z} & =\left(-k_{2} / m\right) v_{z}-g(\cos \theta-1)-v_{x} \omega+(1 / m) u_{1}, \\
\dot{\omega} & =\left(-k_{3} / J\right) \omega+(h / J) u_{2} .
\end{aligned}
$$

Control $u_{1}$ corresponds to the body vertical force minus gravity, while $u_{2}$ corresponds to coupled forces on the wingtips with a net horizontal component. The other forces depend upon the constants $k_{i}$, which parameterize a linear damping force, and $g$, the gravity constant. The constant $h$ is the distance from the center of mass to the wingtip, while $m$ and $J$ are mass and moment of inertia, respectively.

The PVTOL aircraft model fulfills the controllability Assumption 7.1. We design control inputs to track a desired trajectory $\left(x^{d}(t), z^{d}(t), \theta^{d}(t)\right)$ as

$$
\begin{aligned}
u_{1}= & \frac{J}{h} \ddot{\theta}^{d}+\frac{k_{3}}{h} \dot{\theta}^{d}-\frac{\sqrt{2}}{\epsilon} \cos \left(\frac{t}{\epsilon}\right), \\
u_{2}= & \frac{h}{J}-f_{1} \sin \theta^{d}+f_{2} \cos \theta^{d} \\
& \quad-\frac{J \sqrt{2}}{h \epsilon}\left(f_{1} \cos \theta^{d}+f_{2} \sin \theta^{d}\right) \cos \left(\begin{array}{c}
t \\
\epsilon
\end{array}\right),
\end{aligned}
$$



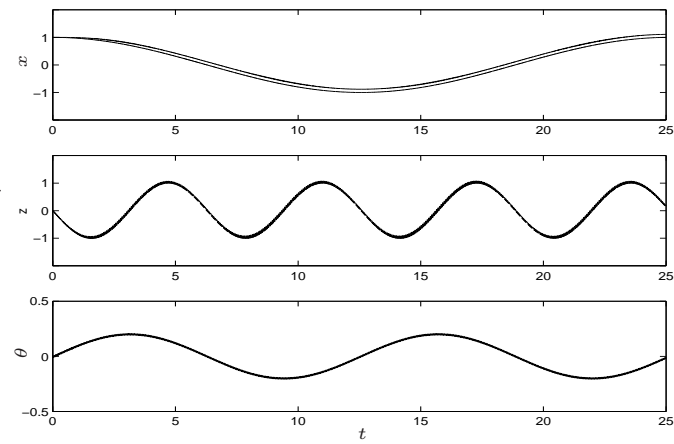

Figure 7: Trajectory design for the PVTOL aircraft model $(\epsilon=.01)$

where we let $c=\frac{J}{h} \ddot{\theta}^{d}+\frac{k_{3}}{h} \dot{\theta}^{d}$ and

$$
\begin{aligned}
f_{1} & =m \ddot{x}^{d}+\left(k_{1} \cos ^{2} \theta^{d}+k_{2} \sin ^{2} \theta^{d}\right) \dot{x}^{d} \\
& +\frac{\sin \left(2 \theta^{d}\right)}{2}\left(k_{1}-k_{2}\right) \dot{z}^{d}+m g \sin \theta^{d}-c \cos \theta^{d}, \\
f_{2} & =m \ddot{z}^{d}+\frac{\sin \left(2 \theta^{d}\right)}{2}\left(k_{1}-k_{2}\right) \dot{x}^{d}+\left(k_{1} \sin ^{2} \theta^{d}\right. \\
& \left.+k_{2} \cos ^{2} \theta^{d}\right) \dot{z}^{d}+m g\left(1-\cos \theta^{d}\right)-c \sin \theta^{d} .
\end{aligned}
$$

The simulations are run with $m=20, J=10, h=5$, $k_{1}=12, k_{2}=11, k_{3}=10, g=9.8$. Figure 7 shows an example of the behavior of these controls.

\section{Conclusions}

We conclude by briefly mentioning the numerous results on mechanical control systems on Riemannian manifolds that are not presented here for reasons of brevity. These include work on optimal control [16, 32, 42], static and dynamic feedback linearization [5, 46], passivity $[20,40]$, energy-shaping methods studied for example in $[8,22,44]$, and small-amplitude algorithms for motion planning and stabilization $[15,37,47]$. An avenue for interesting future research is to relate these areas to the broad problem of geometric trajectory design for mechanical systems.

\section{References}

[1] R. Abraham, J. E. Marsden, and T. S. Ratiu. Manifolds, Tensor Analysis, and Applications, volume 75 of Applied Mathematical Sciences. Springer Verlag, New York, 2 edition, 1988. ISBN 0387967907.

[2] H. Arai, K. Tanie, and N. Shiroma. Nonholonomic control of a three-DOF planar underactuated manipulator.
IEEE Transactions on Robotics and Automation, 14(5): 681-695, 1998.

[3] S. Arimoto. Control Theory of Non-linear Mechanical Systems: A Passivity-Based and Circuit-Theoretic Approach, volume 49 of OESS. Oxford University Press, Oxford, UK, 1996. ISBN 0198562918.

[4] J. Baillieul. Stable average motions of mechanical systems subject to periodic forcing. In M. J. Enos, editor, Dynamics and Control of Mechanical Systems: The Falling Cat and Related Problems, volume 1, pages 1-23. Field Institute Communications, 1993. ISBN 0821892002 .

[5] N. S. Bedrossian and M. W. Spong. Feedback linearization of robot manipulators and Riemannian curvature. Journal of Robotic Systems, 12(8):541-552, 1995.

[6] A. M. Bloch. Nonholonomic Mechanics and Control. Number 24 in Interdisciplinary Texts in Mathematics. Springer Verlag, 2003. ISBN 0387955356.

[7] A. M. Bloch and P. E. Crouch. Nonholonomic control systems on Riemannian manifolds. SIAM Journal on Control and Optimization, 33(1):126-148, 1995.

[8] A. M. Bloch, N. E. Leonard, and J. E. Marsden. Controlled Lagrangians and the stabilization of mechanical systems. I. The first matching theorem. IEEE Transactions on Automatic Control, 45(12):2253-2270, 2000.

[9] F. Bullo. Averaging and vibrational control of mechanical systems. SIAM Journal on Control and Optimization, 41(2):542-562, 2002.

[10] F. Bullo and A. D. Lewis. Kinematic controllability and motion planning for the snakeboard. IEEE Transactions on Robotics and Automation, 19(3):494-498, 2003.

[11] F. Bullo and A. D. Lewis. Low-order controllability and kinematic reductions for affine connection control systems. SIAM Journal on Control and Optimization, 44 (3):885-908, 2005.

[12] F. Bullo and A. D. Lewis. Geometric Control of Mechanical Systems, volume 49 of Texts in Applied Mathematics. Springer Verlag, New York, 2004. ISBN 0387221956.

[13] F. Bullo and K. M. Lynch. Kinematic controllability for decoupled trajectory planning in underactuated mechanical systems. IEEE Transactions on Robotics and Automation, 17(4):402-412, 2001.

[14] F. Bullo and M. Žefran. On mechanical control systems with nonholonomic constraints and symmetries. Systems 8 Control Letters, 45(2):133-143, 2002.

[15] F. Bullo, N. E. Leonard, and A. D. Lewis. Controllability and motion algorithms for underactuated Lagrangian systems on Lie groups. IEEE Transactions on Automatic Control, 45(8):1437-1454, 2000. 
[16] M. Camariña, F. Silva Leite, and P. E. Crouch. Splines of class $\mathrm{C}^{k}$ on non-Euclidean spaces. IMA Journal of Mathematical Control \& Information, 12:399-410, 1995.

[17] J. Cortés. Geometric Control and Numerical Aspects of Nonholonomic Systems, volume 1793 of Lecture Notes in Mathematics. Springer Verlag, New York, 2002. ISBN 3540441549 .

[18] J. Cortés, S Martínez, J. P. Ostrowski, and K. A. McIsaac. Optimal gaits for dynamic robotic locomotion. International Journal of Robotics Research, 20(9):707728, 2001.

[19] P. E. Crouch. Geometric structures in systems theory. IEE Proceedings, 128(5):242-252, 1981.

[20] V. Duindam, S. Stramigioli, and J. Scherpen. Passive compensation of nonlinear robot dynamics. IEEE Transactions on Robotics and Automation, 20(3):480487, 2004.

[21] G. Endo and S. Hirose. Study on roller-walker (system integration and basic experiments). In IEEE Int. Conf. on Robotics and Automation, pages 2032-7, Detroit, MI, May 1999.

[22] K. Fujimoto and T. Sugie. Canonical transformation and stabilization of generalized Hamiltonian systems. In IFAC Symposium on Nonlinear Control Systems, pages 544-549, Enschede, The Netherlands, July 1998.

[23] R. Hermann. Differential Geometry and the Calculus of Variations, volume 49 of Mathematics in Science and Engineering. Academic Press, New York, 1968.

[24] P. L. Kapitsa. Dynamical stability of a pendulum when its point of suspension vibrates. In Collected Papers by P. L. Kapitsa, volume II, pages 714-725, London, 1965. Pergamon.

[25] S. D. Kelly and R. M. Murray. Modelling efficient pisciform locomotion for control. International Journal on Robust and Nonlinear Control, 10(4):217-241, 2000.

[26] P. S. Krishnaprasad and D. P. Tsakiris. Oscillations, $S E(2)$-snakes and motion control: A study of the roller racer. Dynamics and Stability of Systems, 16(4):347-397, 2001.

[27] P. S. Krishnaprasad and D. P. Tsakiris. G-snakes: Nonholonomic kinetic chains on Lie groups. In IEEE Conf. on Decision and Control, pages 2955-2960, Lake Buena Vista, FL, December 1994.

[28] G. Lafferriere and H. J. Sussmann. A differential geometric approach to motion planning. In Z. Li and J. F. Canny, editors, Nonholonomic Motion Planning, pages 235-270. Kluwer Academic Publishers, Dordrecht, The Netherlands, 1993. ISBN 0792392752.
[29] A. D. Lewis. Affine connections and distributions with applications to nonholonomic mechanics. Reports on Mathematical Physics, 42(1/2):135-164, 1998.

[30] A. D. Lewis. Simple mechanical control systems with constraints. IEEE Transactions on Automatic Control, 45(8):1420-1436, 2000.

[31] A. D. Lewis. When is a mechanical control system kinematic? In IEEE Conf. on Decision and Control, pages 1162-1167, Phoenix, AZ, December 1999.

[32] A. D. Lewis. The geometry of the maximum principle for affine connection control systems. Preprint, 2000.

[33] A. D. Lewis and R. M. Murray. Configuration controllability of simple mechanical control systems. SIAM Journal on Control and Optimization, 35(3):766-790, 1997.

[34] A. D. Lewis, J. P. Ostrowski, R. M. Murray, and J. W. Burdick. Nonholonomic mechanics and locomotion: the snakeboard example. In IEEE Int. Conf. on Robotics and Automation, pages 2391-2400, San Diego, CA, May 1994.

[35] K. M. Lynch, N. Shiroma, H. Arai, and K. Tanie. Collision-free trajectory planning for a 3-DOF robot with a passive joint. International Journal of Robotics Research, 19(12):1171-1184, 2000.

[36] D. Manocha and J. F. Canny. Efficient inverse kinematics for general $6 \mathrm{R}$ manipulators. IEEE Transactions on Robotics and Automation, 10(5):648-657, 1994.

[37] S. Martínez and J. Cortés. Motion control algorithms for simple mechanical systems with symmetry. Acta Applicandae Mathematicae, 76(3):221-264, 2003.

[38] S. Martínez, J. Cortés, and F. Bullo. Analysis and design of oscillatory control systems. IEEE Transactions on Automatic Control, 48(7):1164-1177, 2003.

[39] S. Martínez, J. Cortés, and F. Bullo. A catalog of inversekinematics planners for underactuated systems on matrix Lie groups. In IEEE/RSJ Int. Conf. on Intelligent Robots 8 Systems, pages 625-630, Las Vegas, NV, October 2003.

[40] M. C. Muñoz-Lecanda and F.J. Yániz. Dissipative control of mechanical systems: a geometric approach. SIAM Journal on Control and Optimization, 40(5):1505-1516, 2002 .

[41] R. M. Murray, Z. X. Li, and S. S. Sastry. A Mathematical Introduction to Robotic Manipulation. CRC Press, Boca Raton, FL, 1994. ISBN 0849379814.

[42] L. Noakes, G. Heinzinger, and B. Paden. Cubic splines on curved spaces. IMA Journal of Mathematical Control E Information, 6(4):465-473, 1989. 
[43] R. Ortega, A. Loria, P. J. Nicklasson, and H. SiraRamirez. Passivity-Based Control of Euler-Lagrange Systems: Mechanical, Electrical and Electromechanical Applications. Communications and Control Engineering. Springer Verlag, New York, 1998. ISBN 1852330163.

[44] R. Ortega, A. J. van der Schaft, I. Mareels, and B. Maschke. Putting energy back in control. IEEE Control Systems Magazine, 21(2):18-33, 2001.

[45] J. P. Ostrowski and J. W. Burdick. The geometric mechanics of undulatory robotic locomotion. International Journal of Robotics Research, 17(7):683-701, 1998.

[46] M. Rathinam and R. M. Murray. Configuration flatness of Lagrangian systems underactuated by one control. SIAM Journal on Control and Optimization, 36(1): 164-179, 1998.

[47] J. Shen. Nonlinear Control of Multibody Systems with Symmetries via Shape Change. PhD thesis, The University of Michigan, Ann Arbor, MI, May 2002.

[48] H. J. Sussmann. New differential geometric methods in nonholonomic path finding. In A. Isidori and T. J. Tarn, editors, Systems, Models, and Feedback: Theory and Applications, pages 365-384. Birkhäuser Verlag, Boston, MA, 1992. ISBN 0817636331.

[49] H. J. Sussmann and V. Jurdjevic. Controllability of nonlinear systems. Journal of Differential Equations, 12: 95-116, 1972.

[50] A. J. van der Schaft. L2-Gain and Passivity Techniques in Nonlinear Control. Springer Verlag, New York, 2 edition, 1999. ISBN 1852330732. 